\title{
CORRELATION INEQUALITIES FOR SPIN GLASSES
}

\author{
Pierluigi Contucci ${ }^{\dagger}, \quad$ Joel Lebowitz ${ }^{\ddagger}$ \\ † Dipartimento di Matematica, Università di Bologna, e-mail: contucci@dm.unibo.it \\ $\ddagger$ Department of Mathematics, Rutgers University, e-mail: lebowitz@math.rutgers.edu
}

\begin{abstract}
We prove a correlation type inequality for spin systems with quenched symmetric random interactions. This gives monotonicity of the pressure with respect to the strength of the interaction for a class of spin glass models. Consequences include existence of the thermodynamic limit for the pressure and bounds on the surface pressure. We also describe other conjectured inequalities for such systems.
\end{abstract}




\section{Introduction}

Correlation inequalities have played and continue to play an important role in many areas of statistical mechanics. In addition to describing microscopic structure they also provide information about macroscopic properties: for ferromagnetic spin systems they give monotonicity of the critical temperature, inequalities for critical exponents, etc.

It would clearly be desirable to obtain similar results also for disordered systems, like spin glasses, in which both ferromagnetic and anti-ferromagnetic interactions are present with assigned probabilities. In this note we obtain some correlation type inequalities for such systems. These yield various results usually obtained for ferromagnetic systems from the first GKS inequalities ([Gr, Gr2, KS]), e.g. monotonicity and hence existence of the thermodynamic limit of the pressure, and bounds on the surface pressure. We also discuss intriguing examples of versions of the second type GKS inequalities, but at the moment a general proof is still lacking.

\section{Definitions and Results}

Let $\sigma_{n}= \pm 1, n \in \Lambda \subset \mathbb{Z}^{d}$, and denote by $\Sigma_{\Lambda}$ the set of all $\sigma=\left\{\sigma_{n}\right\}_{n \in \Lambda}$, and $\left|\Sigma_{\Lambda}\right|=2^{|\Lambda|}$. To each $X \subset \Lambda$ we associate the spin product $\sigma_{X}=\prod_{i \in X} \sigma_{i}$ and random variable $J_{X}$. The J's, denoted collectively by $\mathbf{J}$, are a family of independent unit symmetric random variables:

$$
\begin{gathered}
\operatorname{Av}\left(J_{X}\right)=0, \\
\operatorname{Av}\left(J_{X} J_{Y}\right)=\delta_{X, Y},
\end{gathered}
$$

where the $\operatorname{Av}()$ denotes the average over the distributions of the J's. The energy of a configuration $\sigma$ is given by

$$
U_{\Lambda}(\sigma)=-\sum_{X \subset \Lambda} \lambda_{X} J_{X} \sigma_{X}+\bar{U}_{\Lambda}(\sigma)
$$

where $\bar{U}_{\Lambda}$ is a non-random (reference) interaction which we need not specify in any detail for the moment. It can in particular be set equal to zero. The $\left\{\lambda_{X}\right\}$, denoted collec- 
tively by $\lambda$, are auxiliary parameters which are used to tune the strength of the different interactions. Some can be set (at the end) equal to zero.

The probability distribution for specified $\lambda$ and $\mathbf{J}$ is given by $\mu=Z_{\Lambda}^{-1} e^{-U_{\Lambda}}$ with random partition function

$$
Z_{\Lambda}=\sum_{\sigma} e^{-U_{\Lambda}(\sigma)}
$$

The quenched pressure is given by

$$
P_{\Lambda}=\operatorname{Av}\left(\log Z_{\Lambda}\right)
$$

We define the thermal correlations for a given $\mathbf{J}$ by

$$
\omega_{A}=\frac{\sum_{\sigma} \sigma_{A} e^{-U_{\Lambda}(\sigma)}}{\sum_{\sigma} e^{-U_{\Lambda}(\sigma)}} .
$$

The quenched correlations $\bar{\omega}_{A}$ are given by $\operatorname{Av}\left(\omega_{A}\right)$ and we also define (dropping the subscript $\Lambda$ )

$$
\rho_{A}=\operatorname{Av}\left(J_{A} \omega_{A}\right)=\frac{\partial P}{\partial \lambda_{A}},
$$

The pressure (and the $\bar{\omega}_{A}$ ) is clearly an even function of each $\lambda_{X}$ and hence $\rho_{A}$ is an odd function of $\lambda_{A}$ and an even function of $\lambda_{X}, X \neq A$.

Theorem 1 Let $\lambda_{A} \geq 0$, then $\rho_{A} \geq 0$. Consequently the quenched pressure $P$ is monotone increasing with respect to each $\lambda_{A}$. i.e.

$$
\frac{\partial P}{\partial \lambda_{A}}=\rho_{A} \geq 0, \quad \forall A \quad \text { with } \quad \lambda_{A} \geq 0
$$

Remark Theorem 1 immediately implies that the pressure $P(\lambda)$ has a minimum for $\lambda_{A}=0$, for any $A \in \Lambda$. E.g. the pressure of a system with symmetric nearest-neighbor random interactions is smaller than the one with added next nearest neighbor interactions. Another consequence is that in the case when $\bar{U}_{\Lambda}=0$ or containing only one site potentials, e.g. for standard spin glasses, the pressure for periodic boundary conditions is larger than the one with free boundary conditions.

Proof: The function $P$ is symmetric and convex with respect to $\lambda_{A}$, i.e.

$$
\rho_{A}=\frac{\partial P}{\partial \lambda_{A}}=0 \quad \text { for } \quad \lambda_{A}=0 .
$$


and

$$
\frac{\partial^{2} P}{\partial \lambda_{A}^{2}}=\operatorname{Av}\left(J_{A}^{2}\left[1-\omega_{A}^{2}\right]\right) \geq 0 .
$$

The first equality follows from symmetry and the second from the fact that $\omega_{A}^{2} \leq 1$. Consequently from $(2.10)$

$$
\rho_{A}\left(\lambda_{A}^{(1)}\right) \geq \rho_{A}\left(\lambda_{A}^{(2)}\right), \quad \forall \lambda_{A}^{(1)} \geq \lambda_{A}^{(2)} \geq 0
$$

and the theorem follows by chosing $\lambda_{A}^{(2)}=0$ and from (2.9).

Theorem 2 When $\bar{U}_{\Lambda}$ is just a sum of one site potentials, i.e. $\bar{U}_{\Lambda}=\sum_{i \in \Lambda} h_{i} \sigma_{i}$, the pressure $P$ is super-additive, i.e., for all disjoint decompositions $\Lambda=\cup_{h=1}^{K} \Lambda_{h}, \Lambda_{l} \cap \Lambda_{m}=\emptyset$ $l \neq m$

$$
P_{\Lambda} \geq \sum_{h=1}^{K} P_{\Lambda_{h}}
$$

Remark These properties of $P$ are similar to those for the ferromagnetic case [Gr2] with the $\rho_{A} \geq 0$ playing the role of the first GKS inequality. This result generalizes the one obtained for the Gaussian case in $[\mathrm{CG}]$.

Corollary 2.1 Assuming thermodynamic stability,

$$
\operatorname{Av}\left(e^{-U}\right) \leq e^{c|\Lambda|}
$$

the super-additivity provided by Theorem 2 implies, by standard methods, monotonicity and existence of the thermodynamic limit for the pressure with free boundary conditions. In particular

$$
p=\lim _{\Lambda \nearrow \mathbb{Z}^{d}} \frac{P_{\Lambda}}{|\Lambda|}=\sup _{\Lambda} \frac{P_{\Lambda}}{|\Lambda|} .
$$

Remark Decomposing a $d$-dimensional hypercube $\Lambda$ into $(d-1)$-dimensional hypercubes (2.12) immediately implies that the pressure in dimension $d, p^{(d)}$ is an increasing function of $d$

$$
p^{(d+1)} \geq p^{(d)}
$$


Corollary (2.1) admits the following proof: to each partition of $\Lambda$ we associate the interpolating potential for $0 \leq t \leq 1$

$$
U_{\Lambda}(t)=\sum_{h=0}^{K} t_{h} U_{\Lambda_{h}}^{(h)}, \quad \Lambda_{0}=\Lambda,
$$

with $t_{0}=t$ and $t_{h}=(1-t)$ for $1 \leq h \leq K$,

$$
U_{\Lambda_{h}}^{(h)}=-\sum_{X \subset \Lambda_{h}} \lambda_{X} J_{X}^{(h)} \sigma_{X}
$$

and we have dropped the argument $\sigma$ and set $\bar{U}_{n}=0$, for notational convenience. The $J_{X}^{(h)}$ are centered independent unit random variables

$$
\operatorname{Av}\left(J_{X}^{(l)} J_{Y}^{(m)}\right)=\delta_{l, m} \delta_{X, Y}
$$

We define the interpolating partition function

$$
Z_{\Lambda}(t)=\sum_{\sigma \in \Sigma_{N}} e^{-U_{\Lambda}(t)}
$$

and we observe that

$$
Z_{\Lambda}(0)=\prod_{h=1}^{K} Z_{\Lambda_{h}}\left(J^{(h)}\right), \quad Z_{\Lambda}(1)=Z_{\Lambda}(J)
$$

We consider the interpolating pressure

$$
P_{\Lambda}(t):=\operatorname{Av}\left(\ln Z_{\Lambda}(t)\right)
$$

Using (2.20) we get

$$
P_{\Lambda}(0)=\sum_{h=1}^{K} P_{\Lambda_{h}}, \quad P_{\Lambda}(1)=P_{\Lambda} .
$$

Considering $\mathcal{C}_{\Lambda}$ the set of all subsets of $\Lambda$ with non empty intersection with more than one $\Lambda_{h}$ a straightforward computation gives

$$
\frac{d}{d t} P_{\Lambda}(t)=\sum_{X \in \mathcal{C}_{\Lambda}} \lambda_{X}^{2} \rho_{X} \geq 0
$$

where the last inequality comes from theorem 1. Hence (2.20) and (2.23), imply (2.12). 
Theorem 3 For finite range interactions, i.e. $\lambda_{X}=0$ for $|X| \geq r$ (e.g. the nearest neighbor case) the first correction to the leading term of the pressure, $T_{\Lambda}$ defined by:

$$
P_{\Lambda}=p|\Lambda|+T_{\Lambda}
$$

has a non positive value and is of surface size:

$$
c|\partial \Lambda| \leq T_{\Lambda} \leq 0
$$

Remark The result (2.25) is analogous to that for the ferromagnetic case in [FL, FC]. The theorem generalizes to all symmetric distributions of the $J_{X}$ the result obtained for the Gaussian case in [CG2].

Proof: We give the proof for nearest-neighbor interactions, $r=2, U_{\Lambda}(\sigma)=\sum_{b} \lambda_{b} J_{b} \sigma_{b}$ where $b$ represents a bond of the lattice $b=\left(n, n^{\prime}\right),\left|n-n^{\prime}\right|=1$. The extension to the general case is straightforward and only changes the value of the constant of (2.25). Given the $d$-dimensional cube $\Lambda,|\Lambda|=L^{d}$ consider its magnification $\left|\Lambda_{k}\right|=(k L)^{d}$. Clearly $\Lambda_{k}$ can be partitioned into $k^{d}$ disjoint cubes $\Lambda_{s}$ all congruent to $\Lambda$. We call $\mathcal{C}_{\Lambda}$ the set of bonds connecting the different $\Lambda_{s}$. In finite volume and with free boundary conditions we have by definition

$$
P_{\Lambda}=\operatorname{Av}\left(\ln Z_{\Lambda}\right)=k^{-d} \operatorname{Av}\left(\ln Z_{\Lambda}^{k^{d}}\right)
$$

Since the limiting pressure per particle is independent of the boundary conditions we have, indicating by $\Pi$ the periodic boundary conditions (see $[\mathrm{FL}]$ )

$$
p|\Lambda|=\lim _{k \rightarrow \infty} k^{-d} \operatorname{Av}\left(\ln Z_{k \Lambda}^{(\Pi)}\right)
$$

By (2.26) and (2.27) we obtain

$$
\begin{aligned}
T_{\Lambda} & =\left(P_{\Lambda}-p|\Lambda|\right) \\
& =\lim _{k \rightarrow \infty} k^{-d} \mathrm{Av}\left(\ln Z_{\Lambda}^{k^{d}}-\ln Z_{k \Lambda}^{(\Pi)}\right) .
\end{aligned}
$$

We chose now the $\lambda$ 's in such a way that

$$
\lambda_{b}= \begin{cases}\lambda, & \text { if } b \in \mathcal{C}_{\Lambda}, \\ 1, & \text { otherwise }\end{cases}
$$


(2.28) then becomes, using the fundamental theorem of calculus,

$$
T_{\Lambda}=\lim _{k \rightarrow \infty} k^{-d}\left[P_{\Lambda}^{(\Pi)}(0)-P_{\Lambda}^{(\Pi)}(1)\right]=-\lim _{k \rightarrow \infty} k^{-d} \int_{0}^{1} \frac{d}{d \lambda} P_{\Lambda}^{(\Pi)}(\lambda) d \lambda .
$$

By a simple computation

$$
\frac{d}{d \lambda} P_{\Lambda}^{(\Pi)}(\lambda)=\sum_{b \in \mathcal{C}_{\Lambda}} \rho_{b}^{(\Pi)}(\lambda)=\left|\mathcal{C}_{\Lambda}\right| \rho_{b}^{(\Pi)}(\lambda)
$$

where we have used the translation symmetry over the torus. By the identity

$$
2\left|\mathcal{C}_{\Lambda}\right|=k^{d}|\partial \Lambda|
$$

we obtain

$$
T_{\Lambda}=-\frac{|\partial \Lambda|}{2} \lim _{k \rightarrow \infty} \int_{0}^{1} \rho_{b}^{(\Pi)}(\lambda) d \lambda,
$$

which immediately entails the theorem.

\section{Other Inequalities}

We have seen that Theorem 1 expresses for spin glasses a monotonicity property that entails the same consequences that the first GKS inequality does for ferromagnetic systems. From this perspective it is interesting to examine whether the quantity

$$
\frac{\partial^{2} P}{\partial \lambda_{A} \partial \lambda_{B}}=\operatorname{Av}\left(J_{A} J_{B}\left[\omega_{A B}-\omega_{A} \omega_{B}\right]\right)
$$

has a definite sign when $A \neq B$ (for $A=B, \omega_{A B}=1$ and the sign is positive). We have some evidence based on explicit calculations (work in progress with F.Unguendoli) that for $A \neq B$ with the $J$ 's satisfying (2.2), the sign is negative, but are unable at this time to produce a general proof or find a counter example. A simple computation based on integration by parts shows that such an inequality would imply that the overlap expectations in Gaussian spin glasses would be monotonic non decreasing with the volume, similar to spin expectations in ferromagnetic systems.

Another interesting question is the case where the $J$ 's are not centered random variables but with $\operatorname{Av}\left(J_{A}\right)=\mu_{A}>0$. We then ask whether $\operatorname{Av}\left(\omega_{A}\right) \geq 0$. This is essentially 
the same question as whether $\operatorname{Av}\left(\omega_{A}\right) \geq 0$ when $\bar{U}_{\Lambda}$ is ferromagnetic. In that case $\omega_{A} \geq 0$ when $\Lambda_{X}=0$ for all $X \subset \Lambda$ and it seems very reasonable to expect that this will be preserved when we add symmetric random interactions. Also in this case we have evidence that the inequality hold for specific cases (work in progress with F.Unguendoli) but unfortunately we don't have a general proof or a counter example. It is interesting to note that in a subspace of the random parameter space in which variance and mean of the $J$ 's are chosen to be identical (Nishimori line) a suitable version of the GKS inequalities can be proved with consequences similar to those obtained here for the surface pressure (see $[\mathrm{MNC}, \mathrm{CMN}])$.

Acknowledgments. P.C. thanks A. Van Enter, C.Giardina, S.Graffi, F.Guerra, F.Den Hollander, H.Nishimori, S.Morita and F.Unguendoli for useful discussions. Research supported in part by NSF Grant DMR0442066 and by AFOSR Grant AF-FA9955004.

\section{References}

[Gr] R. B. Griffiths, Correlation in Ising Ferromagnets, Jou. Math. Phys, Vol. 8, 478-483, (1967)

[Gr2] R. B. Griffiths, A proof that the free energy of a spin system is extensive, Jou. Math. Phys, Vol. 5, 1215-1222, (1964)

[KS] D.G.Kelly, S. Sherman: General Griffiths' Inequalities on Correlations in Ising Ferromagnets, Jou. Math. Phys. 9, 466, (1968)

[CG] P.Contucci, S.Graffi, Monotonicity and thermodynamic limit, Jou. Stat. Phys., Vol. 115, Nos. 1/2, 581-589, (2004)

[CG2] P.Contucci, S.Graffi, On the surface pressure for the Edwards-Anderson Model, Comm. Math. Phys. Stat. Phys., Vol. 248, 207-220, (2004) 
[CMN] P.Contucci, S.Morita, H.Nishimori, Surface Terms on the Nishimori Line of the Gaussian Edwards-Anderson Model Journal of Statistical Physics, Vol. 122, N. 2, 303-312, (2006)

[FL] M.Fisher, J.Lebowitz, Asymptotic free energy of a system with periodic boundary conditions, Commun. Math. Phys. 19, 251-272, (1970).

[FC] M.E.Fisher and G.Caginalp, Wall and boundary free energies, Commun. Math. Phys., 56, no. 1, 11-56 (1977)

[MNC] S.Morita, H.Nishimori and P.Contucci, Griffiths Inequalities for the Gaussian Spin Glass, Journal of Physics A: Mathematical and General, Vol 37, L203-L209, (2004) 